$\operatorname{IPPP} / 02 / 15$

$\mathrm{DCPT} / 02 / 30$

12 March 2002

\title{
SECONDARY REGGEONS IN PERTURBATIVE QCD
}

\author{
M.G.Ryskin and A.G.Shuvaev \\ Department of Physics and Institute for Particle Physics Phenomenology, \\ University of Durham, Durham, DH1 3LE \\ and \\ Petersburg Nuclear Physics Institute, \\ Gatchina, St.Petersburg 188300 Russia
}

\begin{abstract}
Using the Kirschner-Lipatov equation as a basis, we study high energy elastic quark-quark amplitudes, which correspond to secondary Reggeons and pion exchange in the double-logarithmic approximation of perturbative QCD.
\end{abstract}

1. The high energy behaviour of elastic scattering amplitudes is described usually in terms of $t$-channel Reggeon pole (and cut) exchange, that is in terms of the singularities in the complex moment $j$-plane (see, for example, [国). The rightmost singularity, the Pomeron, has the quantum numbers of the vacuum and is placed at $j \approx 1$. In the framework of perturbative QCD the corresponding singularity is formed by the two (reggeized) gluon cut and it's properties are given by the BFKL equation [2, 3] (see [4, 5] for more details).

The secondary Reggeon contribution corresponds to an amplitude with quark-antiquark pair $t$-channel exchange. The leading double-logarithmic (DL) asymptotics of this amplitude was calculated in perturbative QCD by Kirschner-Lipatov [6, 7]. It appears that the quark-antiquark cut occurs at

$$
j=\omega_{0}(t)=\sqrt{2 C_{F} \alpha_{S} / \pi}
$$

for the positive signature amplitude. Here $\alpha_{S}$ is the QCD coupling, $C_{F}=$ $\left(N_{c}^{2}-1\right) / 2 N_{c}$ and $N_{c}=3$ is the number of colors. 
In the $N_{c} \rightarrow \infty$ limit, the positive (even) and negative (odd) signature DL amplitudes are degenerate and both are given by the sum of the laddertype Feynman diagrams. For finite $N_{c}=3$, there appear a non-ladder DL contribution to the odd signature amplitude, which shifts the position of the singularity in $j$-plane. However this non-ladder DL contribution is not large; numerically $\omega_{0}^{(-)} \simeq 1.04 \omega_{0}^{(+)}$, while for a reasonable value of $\alpha_{S}=0.25$ the effective intercept $\omega_{0}^{(+)} \simeq 0.46$. This is close to the phenomenological intercepts of the $\omega, \rho$ (odd signature) and $A_{2}$ (even signature) trajectories $\alpha_{R}(0) \approx 0.5$ which are approximately degenerate. Besides the $\omega, \rho, f$ and $A_{2}$ - Regge poles there are the contributions with other $t$-channel quantum numbers, corresponding to $\pi$ or $A_{1}$ ( unnatural $P$-parity) exchange. In the present paper we discuss the properties of such contributions in the framework of the DL approximation of perturbative QCD.

2. Recall that the DL Kirschner-Lipatov quark-quark amplitude [6] may be written as the Born amplitude $A_{B}$ (shown by continuous lines in Fig.1) multiplied by a DL function $F_{D L}\left(\alpha_{S} \ln ^{2} s /|t|\right)$ which collects the loop contributions caused by the additional gluons. For example, for the simplest colour singlet (in $t$-channel) amplitude, the function $F_{D L}$ reads

$$
F_{D L}^{+}=\frac{I_{1}(2 n)}{n}, \quad n=\sqrt{\frac{C_{F} \alpha_{S}(t)}{2 \pi}} \ln \frac{s}{|t|} .
$$

Thus

$$
A^{+}(s, t)=A_{B} \frac{I_{1}(2 n)}{n},
$$

where

$$
A_{B}=-\frac{4}{9} \cdot 4 \pi \alpha_{S}
$$

The factor $4 / 9$ comes from averaging over the colours of the initial quarks. Since the Bessel function $I_{1}(2 n) \propto e^{2 n}$ at large $n$, the high energy $(\sqrt{s})$ asymptotics of the amplitude (3, 2) takes the form $A^{+} \propto s^{2 n}=s^{\omega_{0}^{(+)}}$.

Note that to DL accuracy, the additional gluons (shown in Fig.1b by the dashed lines) are emitted just by the colour charge of the quark and do not change either the spin structure of the original Born amplitude nor the isospin of the $t$-channel $q \bar{q}$-pair. Therefore, to select the contribution with known quantum numbers in $t$-channel, it is enough to use the Firz relation

$\gamma_{\mu} \times\left.\gamma_{\mu}\right|_{s-\text { channel }} \rightarrow 1 \times 1-\frac{1}{2} \gamma_{\nu} \times \gamma_{\nu}+\frac{1}{2} i \gamma_{\nu} \gamma^{5} \times i \gamma_{\nu} \gamma^{5}-\gamma^{5} \times\left.\gamma^{5}\right|_{t-\text { channel }}$.

As is seen from (41), there is no tensor $\left(\sigma_{\nu \nu^{\prime}}\right)$ component in the $t$-channel expansion of the Born amplitude. From the phenomenological viewpoint we 
have to consider the $A_{2}$-trajectory as the even signature component of amplitude generated by the vector $\left(\gamma_{\nu}\right) t$-channel current. The $\rho$ and $\omega$-trajectories originate from the same vector $\left(\gamma_{\nu}\right)$ current but with odd signature.

At the Born level, relation (14) means that the amplitudes which correspond to natural and unnatural $t$-channel parities (that is $\gamma_{\nu}$ and $\gamma_{\nu} \gamma_{5}$ or 1 and $\gamma_{5}$ terms in (4) ) are degenerate. Of course, besides this amplitude, we have the interaction of quarks mediated by $t$-channel two (and more) gluon exchange, that is by Pomeron (and Pomeron cuts). Moreover the degeneracy between the vector and axial (corresponding to the $\gamma_{\nu} \gamma_{5}$ term in (4) ) amplitudes may be spoiled by higher order loop corrections coming from the region of rather large distances (small quark transverse momenta $k_{t} \leq 1 \mathrm{GeV}$ ), where the effects of confinement are not negligible. This point was discussed in [8]. Contrary to the pure "soft" Regge phenomenological approach (where intercept of the $A_{1}$-trajectory $\alpha_{A_{1}}(0) \leq 0$ [9] is lower than that for the $\rho$-trajectory $\alpha_{\rho}(0) \simeq 0.5$ ) at large scales, the effective (isovector) intercept in the axial $\left(\gamma_{\nu} \gamma_{5}\right)$ channel is expected to be a little larger than that in the vector channel [8], due to the $s$-channel pion contribution coming from the boundary between the perturbative and $k_{t} \leq 1 \mathrm{GeV}$ confinement region .

3. Now let us consider the amplitude $A_{5}$ with pion quantum numbers in $t$-channel. This amplitude originates from the last $\left(\gamma_{5}\right)$ term on the r.h.s. of (四). To avoid the admixture of the $t$-channel gluon state, we choose here the isovector component th. In the Born approximation the high energy spin-flip amplitude is

$$
A_{5}^{B}\left(\lambda=\frac{1}{2}, \lambda^{\prime}=-\frac{1}{2} ;-\frac{1}{2}, \frac{1}{2}\right) \propto \alpha_{s} \frac{t}{s} .
$$

At high orders of $\alpha_{S}$ the amplitude $A_{5}^{B}$ is multiplied by the same DL function $F_{D L}^{(+)}$(目) for even signature exchange (or by the corresponding function $F_{D L}^{(-)}$ for odd signature exchange). Indeed let us consider the first loop diagram Fig.2a. With the help of Firz identity (4), we replace the $\gamma_{\mu} \cdot \gamma_{\mu}$ vertices of the upper gluon in Fig.2a by the effective $\gamma_{5}$ vertex in Fig.2b in order to select the contribution with pion quantum numbers in $t$-channel.

It is convenient to use the light-cone Sudakov variables

$$
k_{\mu}=-\alpha p_{A \mu}+\beta p_{B \mu}+k_{\mu t}, \quad d^{4} k_{\mu}=\frac{s}{2} d \alpha d \beta d^{2} k_{t}, \quad s \simeq 2 p_{A} p_{B},
$$

and to close the contour of the $\alpha$-integral on the lower gluon pole $1 /\left(p_{B}-k\right)^{2}$.

\footnotetext{
${ }^{1}$ It is known that due to the $\gamma_{5}$-anomaly in singlet channel, an admixture of the twogluon state alters strongly the mass and other properties of the $\eta^{\prime}$-meson.
} 
Then the loop integral reads?

$$
\begin{gathered}
C_{F} \frac{g^{2}}{16 \pi^{3}} \int \frac{d^{2} k_{t}}{k^{4}} \frac{s d \beta}{s \beta-k_{t}^{2}} \sum_{\mu=1,2} \bar{u}\left(p_{B}^{\prime}, \lambda^{\prime}\right) \gamma_{\mu}^{\perp} \widehat{k} \gamma_{5} \widehat{k}^{\prime} \gamma_{\mu}^{\perp} u\left(p_{B}, \lambda\right) \\
\simeq C_{F} \frac{\alpha_{S}}{2 \pi} \int \frac{d \beta}{\beta} \frac{d k_{t}^{2}}{k_{t}^{2}} \bar{u} \gamma_{5} u,
\end{gathered}
$$

where within the DL approximation we assume $k^{2} \simeq k_{t}^{2}$ and $k_{t} \gg q_{t}=$ $\left(p_{B}-p_{B}^{\prime}\right)_{t}$; therefore $k_{t}^{\prime} \approx k_{t}$ and $\left(k^{\prime} k\right) \simeq k^{2}$. In addition we account for the fact that the $s$-channel gluon $\left(p_{B}-k\right)$ is on-mass-shell and has only two transverse polarizations $\mu=1,2$ (see [10, 6] for more details).

The interval of the $d \beta$ integration is restricted by the same DL constraint $\beta \gg k_{t}^{2} / s$ as that of the main (vector or axial in $t$-channel) component of the quark-quark amplitude [6, 10]. Thus the first loop correction takes the form

$$
\frac{1}{2} \frac{C_{F} \alpha_{S}}{2 \pi} \ln ^{2} \frac{s}{|t|}=\frac{1}{2} n
$$

which is equal exactly to the corresponding term in the expansion of the DL function $F_{D L}(2)$. The same is valid for the DL contribution from higher loops. As was shown in [6], each higher DL correction does not depend on (or alter) the spin structure of the quark-quark amplitude.

There is an analogous situation for the scalar component (the first term in (4)). Within the DL approximation the scalar and pseudoscalar exchange are degenerate, just as are the vector and axial components, discussed above. 4. Thus at small distances the quark-quark QCD interaction, mediated by the $q \bar{q}$ pair ( $t$-channel) exchange, contains the contributions coming from the vector, axial, scalar and pseudoscalar terms in the Born amplitude. Within the DL approximation these contributions never mix with each other. The higher $\alpha_{S}$ order DL loop corrections simply multiply each term of the Born amplitude by the same function $F_{D L}\left(\sqrt{\alpha_{S}} \ln s /|t|\right) ; F_{D L}^{+}$(or $\left.F_{D L}^{-}\right)$depending on the even (or odd) signature of the amplitude. The 'vector' and 'axial' components conserve the $s$-channel quark helicities, while the 'scalar' and 'pseudoscalar' components flip helicities of the incoming quarks. However these spin-flip amplitudes are suppressed at high energies by one power of $s$; the pion-like, pseudoscalar exchange contains an extra factor $|t| / s$ in comparision with the 'axial' contribution. Recall that the 'axial' ( $A_{1}$-like) component describes the spin-spin correlation, that is the difference of the non-flip amplitudes with different helicities of the incoming quark.

\footnotetext{
${ }^{2}$ We consider here the ladder type loop only as the non-ladder DL correction comes from the pure classical current emission and does not depend on the spin structure of the amplitude at all.
} 
Contrary to the "soft" Regge phenomenology (where the intercepts $\alpha_{A_{1}}(0) \leq 0, \alpha_{\pi}(0) \simeq 0$ of the $A_{1}$ - and $\pi$-trajectories are smaller than intercepts $\alpha_{R}(0) \simeq 0.5$ of a natural parity $\omega, \rho, A_{2}$-trajectories) the perturbative QCD DL amplitudes with natural and unnatural parities are degenerate; the vector $\left(\gamma_{\nu}\right)$ term is degenerate with the axial $\left(\gamma_{\nu} \gamma_{5}\right)$ one, and the scalar term with the pseudoscalar contribution. Therefore at large scales (i.e. small distances) the high energy spin-spin correlation occurs mainly due to the perturbative QCD interaction; the non-perturbative $A_{1}$-exchange dies out with energy. On the other hand the effective intercept, $\omega_{\pi}$, of the perturbative QCD amplitude with pion $t$-channel quantum numbers $\omega_{\pi}=\omega_{0}-1$ is much smaller than $\alpha_{\pi}(0) \simeq 0$. So, for a not too large scale, or $|t|$, the spin-flip (unnatural parity) quark-quark interaction should be described mainly by the non-perturbative pion Regge trajectory.

\section{Acknowledgements}

We are grateful to Prof. J. Bartels who had initiated this work. The work was supported in part by the UK Particle Physics and Astronomy Research Council and by the NATO Collaborate Linkage Grant SA (PST.CLG.976453)5437.

Figure captions

Fig.1 (a) The quark-quark, and, (b) quark-antiquark Born amplitudes. Higher loop corrections are shown in Fig.1(b) by dotted lines.

Fig2. (a) The first loop $q \bar{q}$-amplitude and, (b) the pseudoscalar ( $t$-channel) component of the amplitude .

\section{References}

[1] P.D.B. Collins, Introduction to Regge theory and High Energy Physics, Cambridge University Press, 1977.

[2] L.N. Lipatov, Sov.J.Nucl.Phys. 23 (1976) 338.

V.S. Fadin, E.A. Kuraev, L.N. Lipatov, Sov.Phys.JETP 44 (1976) 443; 45 (1977) 199.

Ya.Ya. Balitskii, L.N. Lipatov, Sov.J.Nucl.Phys. 28 (1978) 822.

[3] L.N. Lipatov, Sov.Phys.JETP 63 (1986) 904.

[4] I.R. Forshaw, D.A. Ross, Quantum Chromodynamics and the Pomeron, Cambridge University Press, 1997. 
[5] L.N. Lipatov, Phys.Rep. 286 (1997) 131.

[6] R. Kirschner, L.N. Lipatov, Nucl.Phys. B213 (1983) 122.

[7] R. Kirschner, Z.Phys. C65 (1995) 505; Z.Phys. C67 (1995) 459.

[8] S.I. Manayenkov, M.G. Ryskin, Eur.Phys.J. C1 (1998) 271.

[9] A.C. Irving, R.P.Worden, Phys.Rep. 34C (1977) 117. G.L. Kane, A. Seidl, Rev.Mod.Phys. 48 (1976) 309.

[10] V.G. Gorshkov, V.N. Gribov, L.N. Lipatov, G.V. Frolov, Sov.J.Nucl.Phys. 6 (1967) 95;

V.G. Gorshkov, Sov.Phys.Uspekhi 16 (1973) 322. 


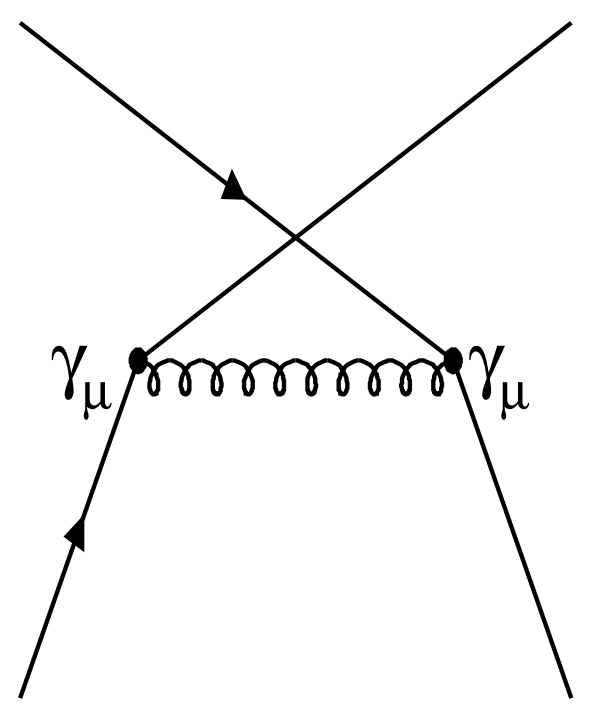

$a$

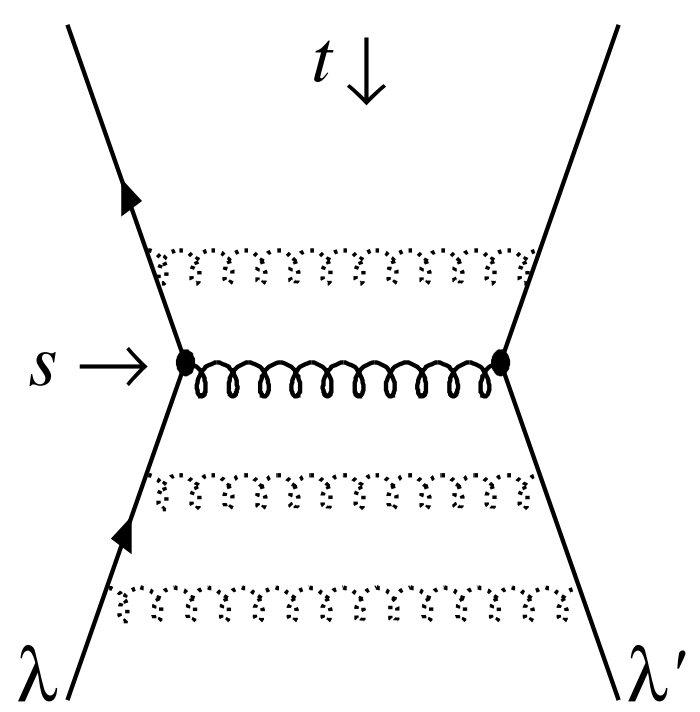

b 


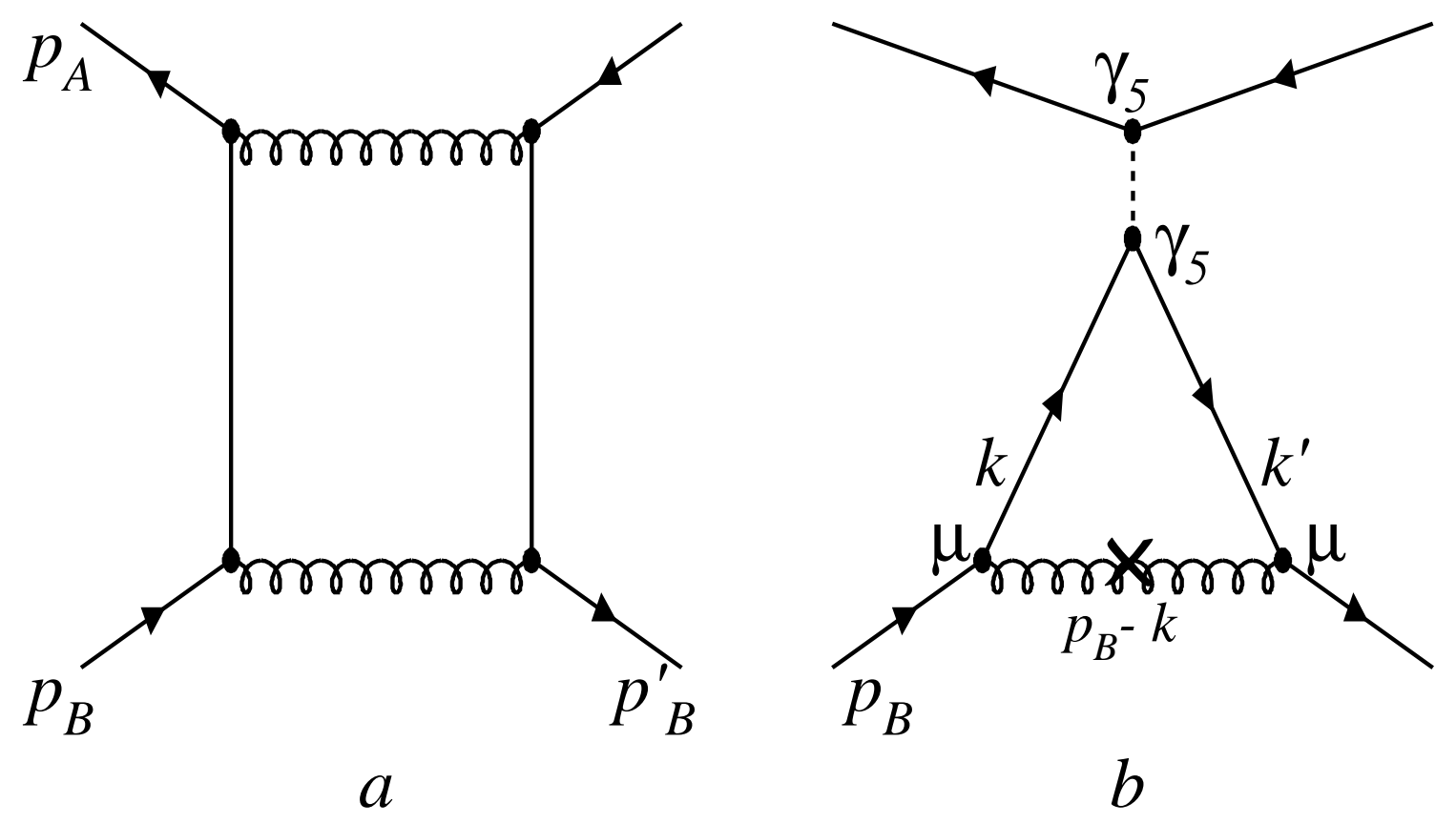

